\title{
Remarks on the Theory of Cosmological Perturbation
}

\author{
Wenbin Lin \\ Institue of High Energy Physics, Chinese Academy of Science, P.O. Box 918-4, Beijing \\ 100039, P.R. China
}

\begin{abstract}
It is shown that the power spectrum defined in the Synchronous Gauge can not be directly used to calculate the predictions of cosmological models on the large-scale structure of universe, which should be calculated directly by a suitable gauge-invariant power spectrum or the power spectrum defined in the Newtonian Gauge.
\end{abstract}

PACS: 98.80.-k; 98.80.Hw; 98.80.Cq 
It is indispensable to adopt the linearized Einstein equation to solve the problem of the evolution of density perturbation in the expanding universe, and this involves the freedom of gauge. Currently, the density perturbation is widely calculated in the Synchronous Gauge $(S G)$ frame and then directly compared with the observations of the large-scale structure of universe, e.g., the abundance and distribution of the galaxies or the clusters (e.g., Refs. [1 5]). Because the power spectrums of density perturbation differ dramatically for small wavenumbers in different gauges, this raises an important problem: Does the density perturbation in the $S G$ frame characterize directly the large-scale structure of universe which is relative to the unperturbed expanding background of universe? I do not think so.

Because the density perturbations defined in different gauges are generally different from each other, we need to construct some suitable gauge-invariant variables that can be directly confronted with the large-scale structure of universe. The gauge-invariant approach to the gravitational perturbation, which was pioneered by Bardeen [6], has been applied to the cosmological perturbation (e.g., Refs. [7],8]). However, there exist some errors in the comparison between the theoretical calculations and the observations of the large-scale structure of universe.

The most general form of the line element for a spatially flat background and scalar metric perturbations can be written as [8]

$$
d s^{2}=a^{2}\left\{(1+2 \Psi) d \tau^{2}-2 B_{\mid i} d x^{i} d \tau-\left[\delta_{i j}-2\left(\Phi \delta_{i j}-E_{\mid i j}\right)\right] d x^{i} d x^{j}\right\}
$$

where $a$ and $\tau$ are the conformal cosmic expansion scale factor and the conformal cosmic time; "I" denotes the background three-dimensional covariant derivative. The corresponding perturbed energy-momentum tensor $T_{\nu}^{\mu}$ has the form

$$
\begin{aligned}
& T_{0}^{0}=\rho(1+\epsilon), \\
& T_{0}^{i}=(\rho+p) U_{\mid i}, \\
& T_{i}^{0}=-(\rho+p)(U-B)_{\mid i}, \\
& T_{j}^{i}=-p(1+\varepsilon) \delta_{j}^{i}-(\rho+p) \Sigma_{\mid i j},
\end{aligned}
$$


here $\rho$ and $p$ are unperturbed density and pressure; $U$ and $\Sigma$ determine velocity perturbation and anisotropic shear perturbation, respectively; $\epsilon$ and $\varepsilon$ denote the fluctuation for density and pressure respectively.

In many gauge-invariant approaches, the gauge-invariant density perturbation was constructed by [6.7]

$$
\epsilon_{m} \equiv \epsilon+3(1+w) \frac{\dot{a}}{a}(U-B)
$$

where a dot denotes the derivative with respect to the conformal time $\tau ; w=p / \rho$ is the ratio of the pressure to the density of background. $\epsilon_{m}$ coincides with the density perturbation $\epsilon_{(C T G)}$ in the Comoving Time-orthogonal Gauge $(C T G$, in which $U=B=0)$, which denotes the density perturbation relative to the spacelike hypersurface which represents the matter local rest frame everywhere [6]. This quantity also coincides with the density perturbation $\epsilon_{(S G)}$ in the Synchronous Gauge (in which $\Psi=B=0$ ) for the pressureless matter system. In other words, $\epsilon_{m}$ denotes the density perturbation relative to the observers everywhere comoving with the matter (not with the unperturbed expanding background of universe because the matter have local velocity other than the Hubble flow). So, $\epsilon_{m}$ has physical significance only for the small-scale perturbation everywhere, and this can be also derived from following facts: The relation between $\epsilon_{m}$ and the general gravitational potential $\phi$ (which was correctly constructed in literatures, see Eq.(6) below) obeys Poisson equation [6.7]:

$$
\nabla^{2} \phi=-k^{2} \phi=4 \pi G \rho a^{2} \epsilon_{m}
$$

where $k$ is the (comoving) wavenumber of Fourier mode. Poisson equation is valid only in the case that the investigated scale is small compared to the Hubble radius $1 / H$ with $H=\dot{a} / a^{2}$ being Hubble constant $\left[\right.$ [1]. Hence, $\epsilon_{m}$ can not be regarded as the physical density perturbation directly for the large-scale (comparable to or larger than the Hubble radius)

\footnotetext{
${ }^{1}$ In the standard big-bang model, the Hubble radius is the same order as the horizon, but in the
} 
perturbation mode in the expanding universe because the gravitational potential $\phi$ has been constructed correctly. Another fact is that the linearized Einstein equation shows that $\epsilon_{m}$ keeps on growing even for the perturbation modes outside both the Hubble radius and the horizon [6, ]], which also means that $\epsilon_{m}$ can not directly characterize the physical large-scale perturbation modes. Therefore, the physical density perturbation which comprises both the small-scale and the large-scale modes can not be described directly by $\epsilon_{m}$, though it is a gauge-invariant quantity.

In fact, the large-scale density perturbation has physical significance only in the case that the corresponding density perturbation everywhere is relative to the unperturbed expanding background of universe. So we must construct a proper gauge-invariant variable to describe the physical density perturbation directly, and then confront it with the large-scale structure of universe. It is my viewpoint that the gauge-invariant quantity constructed by [6],

$$
\epsilon_{g} \equiv \epsilon+\frac{\dot{\rho}}{\rho}(B-\dot{E})=\epsilon-3(1+w) \frac{\dot{a}}{a}(B-\dot{E})
$$

is just what we sought for. $\epsilon_{g}$ coincides with the density perturbation $\epsilon_{(N G)}$ in the Newtonian Gauge $(N G$, in which $B=E=0)$. Correspondingly, two gauge-invariant scalar potentials $\phi$ and $\psi$, both of which become the same as the gravitational potential in the Newtonian limit, are constructed from metric perturbations [8]:

$$
\begin{aligned}
\phi & \equiv \Phi-\frac{\dot{a}}{a}(B-\dot{E}) \\
\psi & \equiv \Psi+\frac{1}{a} \frac{d}{d \tau}[(B-\dot{E}) a] .
\end{aligned}
$$

inflationary scenario, the horizon can be much large than the Hubble radius. The largest scale in causality is set by the horizon, while the physical perturbation modes outside the Hubble radius will hardly grow as long as the equation of state for the background of universe is unchanged. Notice that the perturbation amplitude for all modes will change if the equation of state of the background changes, e.g., $\phi$ changes during the transition from radiation-domonated era to matter-dominated era $[8]$. 
The gauge-invariant pressure perturbation $\delta p / p$ and velocity perturbation $v_{i}$ are constructed as

$$
\begin{aligned}
\delta p / p & \equiv \varepsilon+\frac{\dot{p}}{p}(B-\dot{E}), \\
v_{i} & \equiv(U-\dot{E})_{\mid i} .
\end{aligned}
$$

The time-time part of the linearized Einstein equation gives [8,9]

$$
\nabla^{2} \phi-3 \frac{\dot{a}}{a}\left(\frac{\dot{a}}{a} \psi+\dot{\phi}\right)=-k^{2} \phi-3 \frac{\dot{a}}{a}\left(\frac{\dot{a}}{a} \psi+\dot{\phi}\right)=4 \pi G \rho a^{2} \epsilon_{g}
$$

From this equation we can see that the relation between the potential $\phi$ and the physical density perturbation $\epsilon_{g}$ does not obey Poisson equation any more for the large-scale perturbation modes. At the same time, it can be deduced from the linearized Einstein equation that $\epsilon_{g}$ hardly grows when the perturbation mode is outside the Hubble radius $(k<a H)$.

Hence, though any gauge can be employed to work for the density perturbation, We should adopt the gauge-invariant variable which characterizes the density perturbation relative to the unperturbed expanding background of universe to compare with the large-scale structure of universe directly (here I do not consider the biasing issue).

For the multi-component system, $\epsilon_{g}, \delta p / p$ and $v_{i}$ denote the gauge-invariant variables of total density perturbation, total pressure perturbation and total velocity perturbation respectively, and the corresponding gauge-invariant variables for $\alpha$-component are constructed in similar ways [7]:

$$
\begin{gathered}
\epsilon_{\alpha g} \equiv \epsilon_{\alpha}+\frac{\dot{\rho}_{\alpha}}{\rho_{\alpha}}(B-\dot{E}) \\
(\delta p / p)_{\alpha} \equiv \varepsilon_{\alpha}+\frac{\dot{p}_{\alpha}}{p_{\alpha}}(B-\dot{E}), \\
v_{\alpha i} \equiv\left(U_{\alpha}-\dot{E}\right)_{\mid i} .
\end{gathered}
$$

From above equations we can see that all these gauge-invariant perturbation variables conincide with the corresponding ones in the Newtonian Gauge respectively. We can also 
adopt the density perturbation defined in this gauge to compare with the large-scale structure of universe directly.

Any a real-space fluctuation $\delta(\mathbf{x}, \tau)$ can be decomposed into Fourier modes

$$
\delta(\mathbf{x}, \tau)=\frac{1}{(2 \pi)^{\frac{3}{2}}} \int \delta_{\mathbf{k}}(\tau) e^{i \mathbf{k} \cdot \mathbf{x}} d^{3} k,
$$

with the reality condition $\delta_{\mathbf{k}}^{*}=\delta_{-\mathbf{k}}$. The power spectrum is defined as the mean square of the corresponding Fourier-mode amplitude:

$$
P_{\delta}(k, \tau)=<\left|\delta_{\mathbf{k}}(\tau)\right|^{2}>\equiv|\delta(k, \tau)|^{2}
$$

All the statistical properties of the Gaussian random field can be determined by the power spectrum completely, and for simplicity we only consider this case here. At the same time, we are only interested in the fluctuation relative to the unpertured background everywhere which can be confronted with the large-scale structure of universe directly. The root mean square (rms) fluctuation and two-point correlation function of density on the scale $R$ can be calculated by the physical power spectrum $P_{\epsilon_{g}}(k, \tau) \equiv\left|\epsilon_{g}(k, \tau)\right|^{2}$ directly

$$
\begin{aligned}
\sigma_{\epsilon_{g}}(R, \tau) & =\left[\frac{1}{2 \pi^{2}} \int_{0}^{\infty} P_{\epsilon_{g}}(k, \tau) W^{2}(k R) k^{3} \frac{d k}{k}\right]^{\frac{1}{2}}, \\
\xi_{\epsilon_{g}}(R, \tau) & =\frac{1}{2 \pi^{2}} \int_{0}^{\infty} P_{\epsilon_{g}}(k, \tau) \frac{\sin k R}{k R} k^{3} \frac{d k}{k}
\end{aligned}
$$

where, $W(x)=\frac{3}{x} j_{1}(x)$ is the window function which truncates the contribution of the power spectrum of large wavenumbers to the rms fluctuation (here $j_{1}$ is the first-order spherical Bessel function). When calculating $\sigma_{\epsilon_{g}}(R, \tau)$ or $\xi_{\epsilon_{g}}(R, \tau)$, we need to integrate the power spectrum over all wavenumbers, not merely the modes within the Hubble radius (see Eq.(15)). Because the spectrum $P_{\epsilon_{m}}(k, \tau) \equiv\left|\epsilon_{m}(k, \tau)\right|^{2}$ differs dramatically from $P_{\epsilon_{g}}(k, \tau)$ for small wavenumbers (e.g., $k \leq a H)$, generally we can not replace $P_{\epsilon_{g}}(k, \tau)$ with $P_{\epsilon_{m}}(k, \tau)$ in Eq.(15) directly to obtain the rms fluctuation and correlation function of the density.

The temperature fluctuation of the cosmic microwave background (CMB) can be characterized by the angular power spectrum $C_{l}$. Based on the line-of-sight-integral method developed by Seljak and Zaldarriaga [10], we have: 


$$
C_{l}=\frac{1}{2 \pi} \int_{0}^{\infty} \frac{d k}{k} k^{3}\left[\int_{0}^{\tau_{0}} \Theta(k, \tau) j_{l}\left(k \tau_{0}-k \tau\right) d \tau\right]^{2}
$$

with

$$
\begin{aligned}
\Theta(k, \tau)= & g\left(\frac{1}{4} \epsilon_{\gamma}+\phi+\frac{\dot{v_{b}}}{k}+\frac{\dot{g}}{g} \frac{v_{b}}{k}\right)+e^{-\kappa}(\dot{\phi}+\dot{\psi}) \\
& +g\left(\frac{\Pi}{4}+\frac{3 \ddot{\Pi}}{4 k^{2}}\right)+\dot{g} \frac{3 \dot{\Pi}}{4 k^{2}}+\ddot{g} \frac{3 \Pi}{4 k^{2}}
\end{aligned}
$$

where $j_{l}$ is the spherical Bessel function; $\epsilon_{\gamma}$ is the photon density perturbation; $\Pi$ is a gaugeinvariant quantity denoting the effect of polarization and anisotropic stress of photons; $v_{b}$ denotes the velocity perturbation of baryons; $\tau_{0}$ denotes present time; $g$ is the visibility function and $\kappa$ is the total optical depth at time $\tau$.

Now, let us take the standard cold dark matter (SCDM) model as an example to illustrate the prediction differences between the conventional treatment and my viewpoint. The SCDM model (the cold dark matter density parameter $\Omega_{c}=0.95$, the baryon density parameter $\Omega_{b}=0.05, H_{0}=100 h \mathrm{~km} \mathrm{~s}^{-1} M p c^{-1}$ with $\left.h=0.5\right)$ assumes that the primordial density spectrum in the $S G$ frame $P_{\epsilon(S G)}\left(k, \tau_{i}\right)$ is the Harrizon-Zel'dovich (HZ) spectrum:

$$
P_{\epsilon(S G)}\left(k, \tau_{i}\right) \equiv\left|\epsilon_{(S G)}\left(k, \tau_{i}\right)\right|^{2}=A k
$$

here $A$ is a constant and $\tau_{i}$ is the primordial time. In the conventional treatment, the density perturbation $\epsilon_{(S G)}$ in the $S G$ frame was directly regarded as the physical density perturbation, so the rms fluctuation of the density on the scale of $r_{8}=8 h^{-1} M p c$ was directly corresponded to $\sigma_{\epsilon(S G)}\left(r_{8}, \tau_{0}\right)$, which is related to $P_{\epsilon(S G)}\left(k, \tau_{0}\right) \equiv\left|\epsilon_{(S G)}\left(k, \tau_{0}\right)\right|^{2}$ by

$$
\sigma_{\epsilon(S G)}\left(r_{8}, \tau_{0}\right)=\left[\frac{1}{2 \pi^{2}} \int_{0}^{\infty} P_{\epsilon(S G)}\left(k, \tau_{0}\right) W^{2}\left(k r_{8}\right) k^{3} \frac{d k}{k}\right]^{\frac{1}{2}} .
$$

In the foregoing discussions we have shown that this picture is wrong because $\epsilon_{(S G)}(k, \tau)$ (or its spectrum $P_{\epsilon(S G)}(k, \tau) \equiv\left|\epsilon_{(S G)}(k, \tau)\right|^{2}$ ) can not characterize the physical density fluctuation directly. The physical density spectrum corresponding to the assumption of the SCDM model on the primordial density spectrum should be

$$
P_{\epsilon_{g}}(k, \tau)=P_{\epsilon_{g}}\left(k, \tau_{i}\right) T_{\epsilon_{g}}^{2}(k, \tau) \simeq\left(\frac{3 a_{i}^{2} H_{i}^{2}}{k^{2}}\right)^{2} A k T_{\epsilon_{g}}^{2}(k, \tau),
$$


where, the subscript " $i$ " denotes the primordial time; $P_{\epsilon_{g}}\left(k, \tau_{i}\right) \simeq\left(3 a_{i}^{2} H_{i}^{2} / k^{2}\right)^{2} A k$ is the physical primordial spectrum corresponding to the $\mathrm{HZ}$ spectrum in the $S G$ frame (notice that all the interesting modes are far outside the Hubble radius at time $\tau_{i}$, i.e., $k<<a_{i} H_{i}$ ), and $T_{\epsilon_{g}}(k, \tau) \equiv \frac{\epsilon_{g}(k, \tau)}{\epsilon_{g}\left(k, \tau_{i}\right)}$ is the transfer function of physical density spectrum due to the evolution of density perturbation from the primordial time $\tau_{i}$ to the time $\tau$.

Fig.1 shows the physical density spectrum $P_{\epsilon_{g}}(k, \tau)$ of the SCDM model at the redshift $z=0$ and $z=10$, with the corresponding $P_{\epsilon(S G)}(k, \tau)$. It can be seen that $P_{\epsilon(S G)}(k, \tau)$ can approximate the physical spectrum only for the large wavenumbers (small-scale modes), and differs significantly from the latter for the wavenumbers outside the Hubble radius. By the way, $P_{\epsilon(S G)}(k, \tau)$ differs hardly from the gauge-invariant power spectrum $P_{\epsilon_{m}}(k, \tau)$ for the SCDM model in which the universe was dominated by the pressureless matter at very high redshift. In the conventional treatment, only the power spectrum of the wavenumbers inside the Hubble radius is taken seriously, and the part outside the Hubble radius is thought to have no physical significance. This viewpoint is not correct: When a real-space fluctuation is decomposed into Fourier modes, all these modes will have physical significance whether or not they are inside the Hubble radius (see Eq.(13)). Only the whole power spectrum can determine a real-space Gaussian fluctuation completely. In fact, the power spectrum of the wavenumbers outside the Hubble radius has also been included in the current calculation of the density fluctuations (e.g., see Eq.(19)) and the angular power spectrum $C_{l}$ for CMB (see Eq.(16)). So, the conventional treatment is inconsistent upon this point. More important, the temperature fluctuation detected by the COBE satellite (e.g., $C_{2}$ ) is dominated by the power spectrum of the wavenumbers outside the Hubble radius at the recombination era! On the other hand, inflationary scenario has provided a well-known mechanism of the nonvanishing power spectrum for the wavenumbers outside the Hubble radius.

Fig.1 also reveals another important difference between the physical spectrum and $P_{\epsilon(S G)}(k, \tau)$ : the shape of the physical spectrum changes even for the pressureless matter system, because the physical density perturbation modes far outside the Hubble radius can not grow due to the causality; while the shape of $P_{\epsilon(S G)}(k, \tau)$ for the SCDM model 
hardly changes, because the growth rate of $\epsilon_{(S G)}(k, \tau)$ is independent of wavenumber $k$ for the presureless system.

Now we re-check some predictions of the SCDM model. From Eqs.(15) and (20) we find that the rms fluctuation $\sigma_{\epsilon_{g}}(R, \tau)$ and the correlation $\xi_{\epsilon_{g}}(R, \tau)$ of density are both divergent on any scale $R$ at any time $\tau$, so do those of the potential $\phi$, if the physical density spectrum has the HZ shape exactly in the limit of $k \rightarrow 0$ in the $S G$ frame. In fact, these unreasonable predictions caused by the divergent spectrum have also been reflected in the calculations of the temperature fluctuation of CMB, though it is very implicit. From the combination of Eqs.(16), (17) and (20) we can obtain that $C_{0}$ is divergent too. This means that the temperature fluctuation of $\mathrm{CMB}$ is infinite in any direction. Because people are only interested in the angular distribution of the temperature anisotropy of CMB (e.g., $C_{l}$ for $l \geq 1$ ), this unreasonable prediction of the SCDM model has been ignored so far.

All these unreasonable predictions are due to the fact that the power spectrum was not cut off effectively. Any physical power spectrum should become negligible as wavenumber $k$ tends to zero due to the causality. So, the primordial spectrum should be cut off effectively at a minimum wavenumber $k_{m i n}$, e.g., $a / k_{\text {min }}$ is at most the same order as the horizon (notice that the horizon may be much larger than the Hubble radius). After doing so, we can see that all calculation results are convergent and dependent on $k_{\min }$.

The key point lies in how to determine the effective minimum wavenumber $k_{\min }$ for the power spectrum. If $k_{\text {min }}$ is inside the Hubble radius at any time, there will not be so many problems to discuss here. However, $k_{\text {min }}$ can be far outside the Hubble radius (for example, the quantum fluctuation in the early universe can be stretched far outside the Hubble radius by inflation process), otherwise we can not explain the temperature fluctuation detected by the COBE satellite, which showed that these fluctuation modes are outside the Hubble radius at the recombination era.

In conclusion, the power spectrums differ dramatically for the modes outside the Hubble radius in different gauges, however, the calculation of the rms density fluctuation needs to integrate the power spectrum for all perturbation modes (see Eq.(15)), not merely the 
modes within the Hubble radius, so do the calculations of the two-point correlation function, the abundance of the galaxies or clusters by Press-Schechter formula, and the angular power spectrum $C_{l}$ for $\mathrm{CMB}$, etc. In the above demonstrations we have shown that these quantities should be calculated directly by the gauge-invariant spectrum $P_{\epsilon_{g}}(k, \tau)$, or the spectrum $P_{\epsilon(N G)}(k, \tau) \equiv\left|\epsilon_{(N G)}(k, \tau)\right|^{2}$ in the Newtonian Gauge which coincides with $P_{\epsilon_{g}}(k, \tau)$, but not directly by the spectrum $P_{\epsilon(S G)}(k, \tau)$ in the Synchronous Gauge. On the other hand, the density fluctuations and the CMB anisotropies are dependent on the cut-off wavenumber $k_{\text {min }}$ of the physical power spectrum. $k_{\text {min }}$ is not set by the Hubble radius simply, but by the physical process in early universe, such as inflation. In the inflationary picture there generally exists a non-vanishing power spectrum for the modes outside the Hubble radius, i.e., $k_{\min }$ can be far outside the Hubble radius! These conclusions will change dramatically the scenarios of the current cosmological models on the formation of the large-scale structure of universe.

I am thankful to R. Brandenberger for reading manuscript and giving valuable comments. I also thank E. Bertschinger for using the COSMICS, and U. Seljak and M. Zaldarriaga for using the CMBFAST. This work was supported in part by the National Natural Science Foundation of China under Grant No.10047004. 


\section{REFERENCES}

[1] Peebles P J E 1980 The Large Scale Structure of the Universe (Princeton Univ. Press, Princeton)

[2] Bardeen J M, Bond J R, Kaiser N and Szalay A S 1986 ApJ 30415

[3] White M, Scott D and Silk J 1994 Ann. Rev. A\&A 32319

[4] Dodelson S, Gates E I and Turner M S 1996 Science 27469

[5] Gawiser E and Silk J 1998 Science 2801405

[6] Bardeen J M 1980 PRD 221882

[7] Kodama H and Sasaki M 1984 Prog. Theo. Phys. Suppl. 781

[8] Mukhanov V F, Feldman H A and Brandenberger R H 1992 Phys. Rep. 215203

[9] Ma C P and Bertschinger E 1995 ApJ 4557

[10] Seljak U and Zaldarriaga M 1996 ApJ 469437 


\section{Captions for Figure}

Fig.1 The physical density spectrum for the SCDM model (denoted as the solid lines), with the corresponding spectrum in the Synchronous Gauge (denoted as the dashed lines). Since the physical rms fluctuation $\sigma_{\epsilon_{g}}\left(r_{8}, \tau_{0}\right)$ (see Eq.(15)) is divergent for the SCDM model, for simplicity the normalization for the density spectrum is chosen so that $\sigma_{\epsilon(S G)}\left(r_{8}, \tau_{0}\right)=1$ (see Eq.(19)) instead; The upper and the lower lines correspond to the cases of $z=0$ and $z=10$, respectively. 


\section{FIGURES}

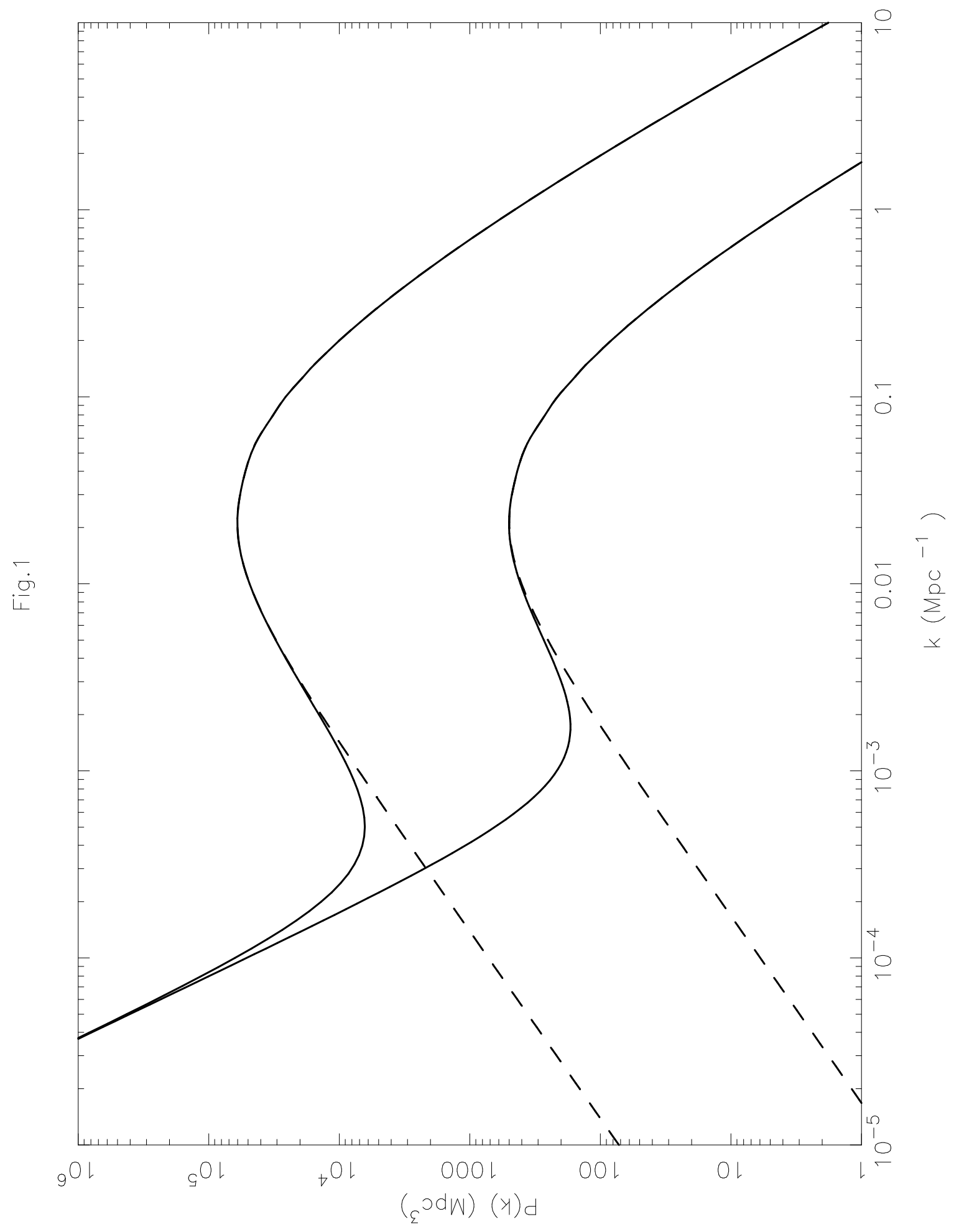

